\title{
Large-scale Residential Demand Response ICT Architecture
}

\author{
Matthias Strobbe $^{1}$, Koen Vanthournout ${ }^{2}$, Tom Verschueren ${ }^{1}$, Wim Cardinaels ${ }^{2}$ and Chris Develder ${ }^{1}$ \\ ${ }^{1}$ Ghent University - iMinds \\ Dept. of Information Technology - IBCN, Ghent, Belgium \\ Email: matthias.strobbe@intec.ugent.be \\ ${ }^{2}$ VITO-EnergyVille \\ Mol, Belgium \\ Email: koen.vanthournout@vito.be
}

\begin{abstract}
The Flemish project Linear is an example of an ongoing large scale residential demand response pilot that aims to validate innovative smart grid applications that exploit the rollout of information and communication technologies (ICT) in the power grid. In this paper, we discuss the design of such a scalable, reliable and interoperable ICT infrastructure that interconnects 245 residential power grid customers with the backend systems of various actors: e.g., energy service providers (ESPs), flexibility aggregators, distribution system operators (DSOs), balancing responsible parties (BRPs). The use cases rolled out in Linear, built on top of our proposed ICT architecture, involve sharing both metering data and flexibility information (esp. for time shifting) of the households, and demand response (DR) algorithms for the balancing of renewable energy and the mitigation of voltage and power issues in distribution grids.
\end{abstract}

Index Terms-Smart Grids, Residential Demand Response, ICT architecture, Pilot

\section{INTRODUCTION}

The power grid is moving away from the current centralized power generation paradigm. With governments promoting local renewable power generation at residential sites, distributed power generation is gaining in popularity. Environmental concerns and efforts to become less dependent on fossil fuels are the driving force for the replacement of traditional energy sources by green alternatives. The EU 20-20-20 targets aim for a reduction in EU greenhouse gas emissions of at least $20 \%, 20 \%$ of EU energy consumption to come from renewable energy sources, and a reduction in energy consumption of $20 \%$ by 2020 [1].

The intermittent nature of renewable energy sources, such as solar or wind, and the fact that these sources are often uncontrollable, makes it difficult to balance demand and supply, which is essential for the correct operation of the power grid. Additionaly, electricity demand is rising, e.g., as a result of the ongoing electrification of the vehicle fleet [2] and the adoption of heat pumps. Also, investments in controllable (fossil-fuel, typically gas-fired) plants are decreasing due to a low profitability as power from renewable energy sources gets priority on the grid.
To cope with intermittent production, more flexibility is needed in the power grid, which can be found partially on the consumer side in terms of consumption that can be shifted in time. Whereas demand response (DR) is increasingly deployed in the industry, the large potential in the residential sector remains until now unused.

In residential settings, other criteria apply: protection of comfort is of prime importance to achieve sustained participation in demand response; individual flexibility sources only contribute a small amount of energy, but they are large in number, requiring relatively large investments in control and communication technologies per unit of flexible power. As such, the technology needed for residential DR is fundamentally different from the industrial equivalent and requires the extended implementation of scalable information and communication technology (ICT) into the power grid domain.

Research and industrial partners joined forces in the Flemish smart grid project Linear ${ }^{1}$, in close collaboration with the government, to develop, deploy and evaluate residential demand response technology in a large-scale pilot with 245 households. Goal of this pilot is investigating user behaviour and acceptance, and to design, develop and evaluate the technology required to bring residential DR business cases to the field.

Linear is not the only pilot on residential demand response. Several other pilots, that include smart automated appliances, have been executed or are ongoing, e.g., [3]-[13]. These projects typically focus on a limited number of types of smart appliances, with smart heating and smart charging of electrical vehicles the most dominant ones. Linear distinguishes itself by integrating one of the widest arrays of different types of appliances. Additionally, most residential DR pilots focus on a single or limited set of control/business cases, and are typically vertically integrated, i.e., each link in the architecture is realized by a single company and/or component. Also here the Linear pilot takes a wider scope by supporting appliances

\footnotetext{
${ }^{1}$ http://www.linear-smartgrid.be/?q=en
} 
from multiple vendors and by supporting multiple energy service providers. This wider scope also translates to the size of the pilot; in terms of number of participants with smart appliances and number of smart appliances per residence, Linear ranks among the most ambitious, which makes the Linear residential DR pilot one of the most complete today.

In this paper we outline the ICT infrastructure that was designed and deployed for the realization of residential demand response in practice. In Section II we provide more details on the setup and goals of the pilot. We outline the requirements (Section III) and provide details about the design and deployment of the architecture (Section IV). In Section V we discuss the current status of the pilot with some first results. Conclusions are stated in Section VI.

\section{LINEAR PILOT}

The pilot for the evaluation of residential demand response services in a realisitic setting was set up in different phases starting with the installation of measurement devices in the houses followed by the installation of remotely controllable smart appliances. The flexibility offered by these smart appliances is used for four different business cases:

- Portfolio Management: Can we make customers shift their energy consumption as much as possible towards off-peak hours and periods with an abundant amount of renewable energy, based on day-ahead predictions?

- Wind Balancing: Can we reduce intra-day imbalance costs for the BRP, caused by the deviation between predicted and produced wind energy?

- Transformer Aging: Can we reduce the aging of transformers by shifting the energy consumption to avoid high transformer temperatures?

- Line Voltage Management: Can we avoid too large voltage deviations in local grids, for example caused by the injection of a high amount of solar energy on sunny summer days?

Residence metering or smart meters, and smart plugs for submetering are deployed to get measurements of household consumption and production ( 94 households have photovoltaic panels representing a total of $400 \mathrm{kWp}$ ) and detailed appliance consumption. In total, about 2000 submetering points are installed and 106 households are equipped with smart meters. The metering equipment is first used to collect reference measurements so that the effect of the experiments on the consumption patterns of the involved households can be assessed. At this moment, during the actual experiments, the consumption and production measurements are used by the control algorithms and to analyse the effectiveness of the DR systems.

The capturing of reference measurements started in 2010 for the first participants and was extended to all users by the end of 2012. In the mean time the smart appliances were installed and from spring 2013 the actual experiments started with remote control of these appliances. These experiments will run until October 2014.
Two types of smart appliances that offer large amounts of flexibility and can be automated were selected to minimize the comfort impact for the participants. The first type consists of postponable appliances, such as dishwashers, washing machines and tumble dryers, 445 of which are deployed in the pilot. Second type are appliances with buffers, such as smart domestic hot water buffers (DHW, 15 deployed) and electrical vehicles (EV, 7 deployed).

Every participant received a home gateway which interacts with the measurement equipment and smart appliances, and a display in the form of a tablet so that they can get insight in their consumption patterns and the amount of provided flexibility (and associated financial bonus).

Users are asked to provide as much flexibility as possible when configuring their smart appliances, for example by configuring these devices before going to work with a deadline at the time they return home.

The total of 245 households are partitioned in two subsets. The first comprises 139 households that are geographically spread over the region of Flanders. These users participate in the experiments with a commercial focus (portfolio management, wind balancing). The second subset of 106 users are living close together in two selected neighbourhoods so that several users are connected to the same feeder and transformer. This allows to test the location specific, technical business cases (transformer aging, line voltage management). In those neighbourhoods also wind balancing is tested to assess the effect on the local distribution grid.

Two types of interaction models are used in the pilot:

- Residential demand response, based on day-ahead dynamic prices [14] is tested with 60 families. A day is divided in 6 fixed blocks (night, morning, noon, afternoon, evening, late evening), but the tariffs are different from day to day and are based on the day ahead power market scaled-up to 2020 wind and solar production predictions. The users can consult these tariffs on their display from 6 PM the day before. If they shift consumption from expensive hours to cheap moments of the day (in comparison with their reference measurements), they get a financial reward. This model supports the business case of day ahead portfolio management.

- The second model is tested with the participants that have smart appliances. The participants get a financial reward based on the amount of flexibility they offer with their appliances. The flexibility is used for all four Linear business cases.

\section{ARCHITECTURE REQUiREMENTS}

Figure 1 gives an overview of the actors involved in residential demand response services in the Linear setup. Realtime energy measurements from the end users are collected via their home gateway and sent to the back-end of the Energy Service Provider (ESP), who can use this data to offer services as energy consumption visualization, energy audits, etc. If smart appliances are present, the home gateway also collects flexibility status information and sends this data via 
the ESP backend or directly to a flexibility aggregator. This actor collects flexibility data from a large number of users and sells it to interested parties, such as BRPs and DSOs, who use the offered flexibility for commercial or technical goals as balancing, congestion \& power quality management, and asset management.

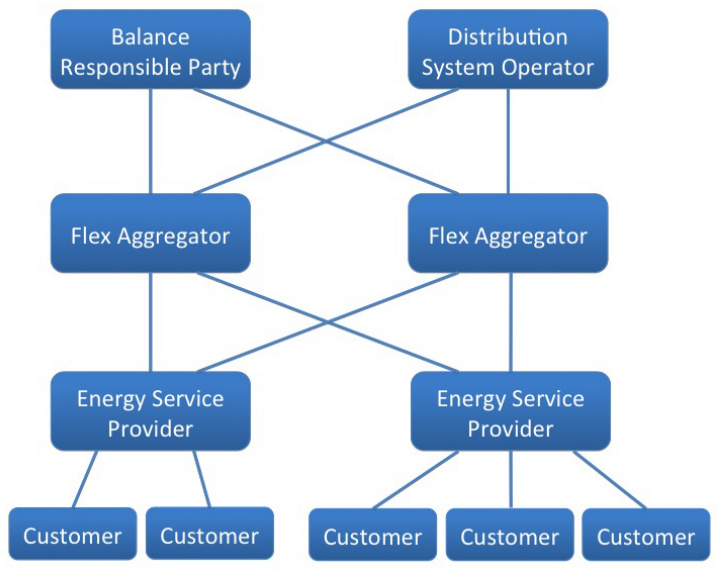

Fig. 1. Relevant roles in the Linear value network for demand response services

During the pilot design phase, based on the value network analysis and in cooperation with the industrial partners, following requirements were identified for the Linear architecture:

- It must be possible to support residential demand response cases for both BRPs and DSOs. The business cases must not run in parallel, but easy switching from one to the other must be supported.

- Smart appliances and measurement devices are connected to and aggregated via a home gateway that is installed in each residence. These are managed by a Energy Service Provider.

- The architecture must support multiple actors of each type (energy service providers, flexibility aggregators, BRPs, DSOs), each with their own proprietary system, the inner workings of which may not be disclosed.

- The status data and commands exchanged with the smart appliances must be as vendor independent as possible.

- As the purpose of the project is a residential demand response pilot, all data measured locally should be centrally collected into a single database to allow in depth analysis of the operation of the system and the interaction with the participants.

- PV installations must be measured, and at least all smart appliances must be submetered. The smart meters of both DSOs particating in the project must be supported. Extra energy measurements at household level must be supported for those cases where no smart meter is available.

The ensuing design consists of three layers, where the abstraction and interfacing in between those levels is key: the smart appliances and measurement equipment level, the energy service provider level and the central control and data acquisition level. A lot of attention has been given to achieve as much as possible device abstraction for the smart appliances (see Section IV-B). Also the interface to the energy service providers and the flex aggregators has been specified in detail.

\section{ICT ARCHITECTURE FOR RESIDENTIAL DEMAND RESPONSE}

\section{A. Overview}

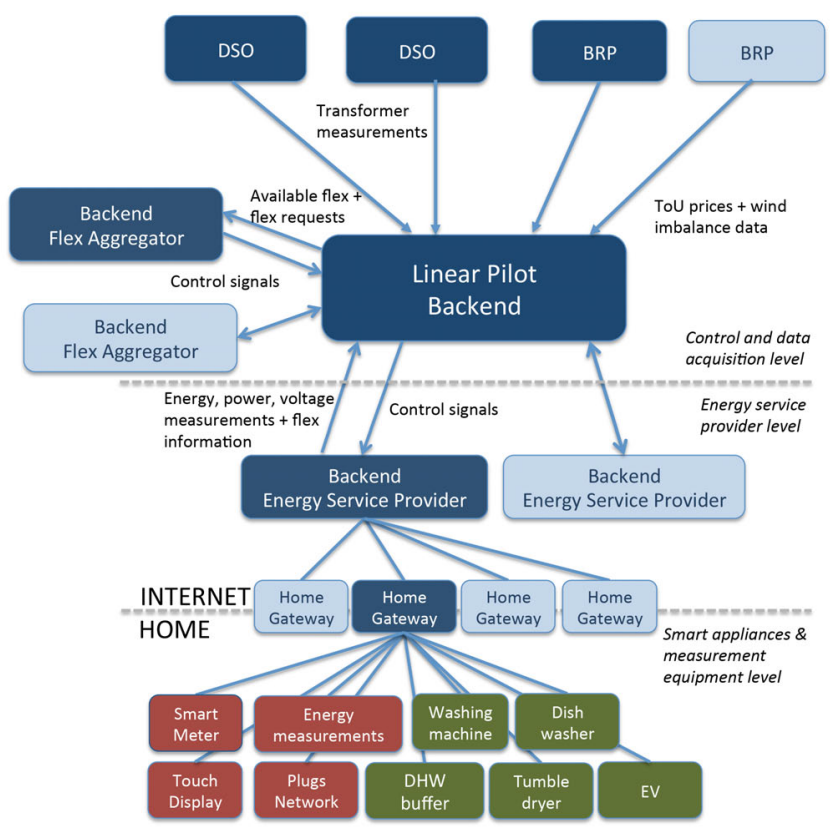

Fig. 2. Overview of the ICT architecture for the Linear pilot. The architecture supports multiple actors of each type. 1 energy service provider, 1 flexibility aggregator, 2 DSOs and 1 BRP participate in the Linear pilot. In the households almost all combinations of measurement equipment and smart appliances occur.

Based on the identified requirements a scalable, reliable and interoperable ICT architecture was designed, implemented and deployed (Figure 2). Every participant received a home gateway from the energy service provider partner, that sends all locally collected consumption, production and flexibility data in real-time to the backend of the energy service provider and from there to the pilot backend. Inputs and requests for using the available flexibility are sent from the BRP and DSO partners in the form of wind imbalance setpoints, dynamic price information and real-time measurements from 5 transformers in the field (current, voltage, phase and temperature).

The pilot backend sends all relevant info to the server of the flex aggregator that tries to optimally match requests for flexibility with the currently available flexibility. The control logic of the flex aggregator will generate control signals (e.g. to start a number of white goods or stop charging an EV) which are sent via the pilot backend and ESP backend to the home gateways of the end users and from there to the smart appliances. Users without smart appliances receive the ToU prices from the pilot backend through the ESP backend and 


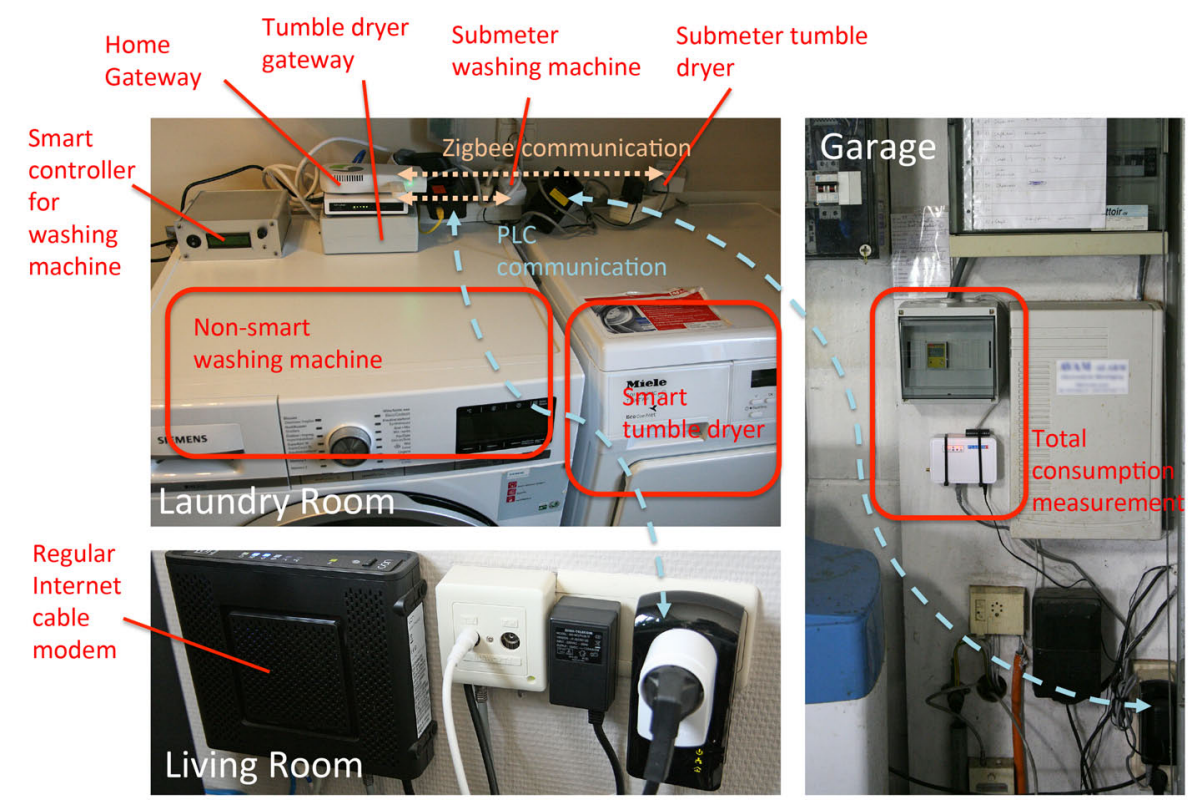

Fig. 3. Overview of an example home setup. This user has 2 smart appliances: a smart tumble dryer that communicates via PLC with the gateway of the manufacturer and from there via Ethernet with the home gateway. The non-smart washing machine is controllable via a dedicated controller developed in the project that also communicates via Ethernet with the home gateway. The smart appliances are submetered via smart plugs using Zigbee to communicate with the home gateway. 8 more smart plugs are installed in the house to submeter other (non-smart) appliances. The total household measurement is communicated over PLC to the home gateway. PLC is also used for the communication between home gateway and regular cable modem to reach the outside world.

in a similar way users can request the status of their financial boni.

\section{B. In-home Communication \& Control}

As stated in Section III, a key requirement of the Linear pilot is device abstraction and, as such, generic interfaces for the smart appliances were the dominant design driver.

The flexibility status of postponable appliances, i.e., dishwashers, washing machines and tumble drivers, is abstracted by a configuration time, a start deadline and an estimate of the program power consumption in function of the time. They take a single control signal, which is the start command. All postponable appliances are assumed to have comfort protection, which implies that the program is started by the appliance if no start signal is received at the start deadline. The Linear consortium contains two home appliance manufacturers. The first supplied the pilot with a smart version of all three postponable appliances. These communicate via power line communication with a gateway of that manufacturer, which connects via Ethernet to the Home Gateway and offers an XML-overHTTP interface. The original interface was extended to include the parameters required by the Linear postponable appliances abstraction. The second manufacturer provided regular nonsmart dishwashers and washing machines for the pilot, which are controlled by an external controller (developed within the project) that provides the required parameters using JSONRPC over HTTP and Ethernet. This means that, although multiple drivers needed to be developed for the Home Energy Gateway, the same status and control signals are supported by all postponable appliances, and once at the level of the Energy
Service Provider, they behave functionally identical. All user configuration is on the smart appliance itself or on the attached controller. Figure 3 gives an example of a setup in one of the Linear households.

The smart DHW buffers support the generic interface defined in [15], also via JSON-RPC over HTTP and Ethernet. Comfort settings are configured using a webpage on the buffer controller. However, no changes to the default settings were ever necessary, which implies that in practice there was no interaction required with the participants for the smart DHW buffer.

The electrical vehicles employ an interface similar to that of the postponable appliances: configuration time, departure time, charging power and charging time. Consumption measurements show that a constant charging power can be assumed for the EV model used in the pilot. A difference with the postponable appliances program is that charging can be interrupted, and so both start and stop commands are provided. No dedicated EV charging infrastructure is deployed in the pilot. Instead, the smart plugs that are used for submetering, and that can be remotely switched, control the charging of the EVs.

\section{Central Pilot Backend}

Important requirements for the central pilot backend are a guaranteed availability so that no data is lost nor an experiment is interrupted, a secure storage and access to the acquired data, and tools for an efficient analysis and validation of the data in real-time. To meet these requirements an architecture consisting of several stages was designed (Figure 4). 


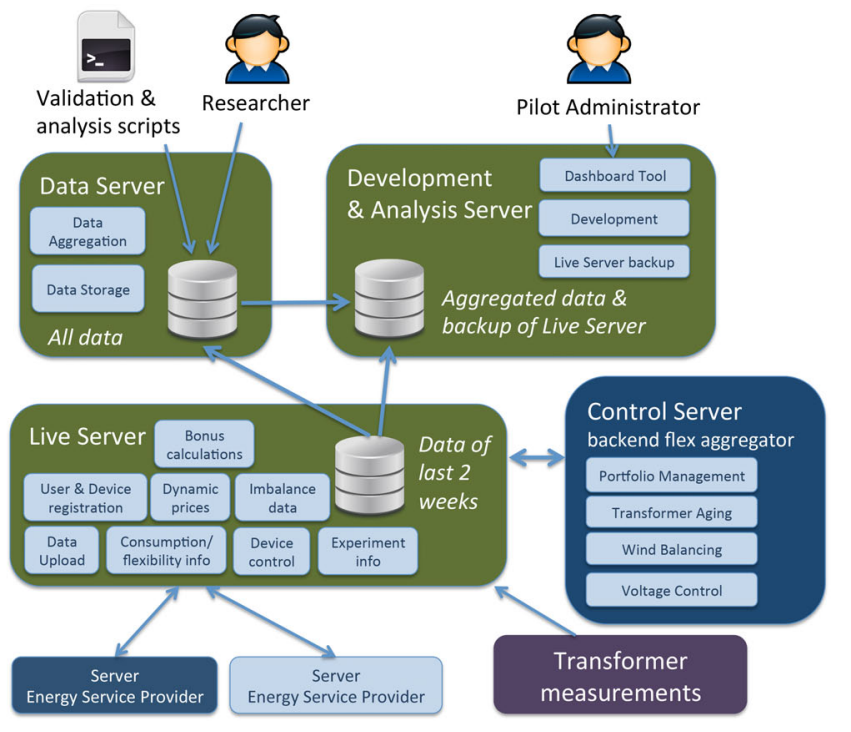

Fig. 4. Overview of the Linear backend.

The Live Server is responsible for the real-time gathering of data, the registration of new users, providing relevant information to the end users (e.g., bonuses) and to the business logic of the flex aggregators (e.g., wind imbalance data), and forwarding of control signals to the participants. Standard web service technology is used for the communication between the backends of the different actors. There is also a direct communication link via GPRS to 5 transformers in the field.

All received data is replicated, in real-time, to the Data Server for validation and detailed analyses of the ongoing and finished experiments. Validation scripts check the correctness of the received data and flag records that are invalid (e.g., extreme values, total consumption values that are smaller than the sum of the submetered appliances, records with incorrect timestamps, etc.). The Data Server is also responsible for filtering and aggregating the received data via scripts, and forwarding it to the Development \& Analysis Server where this data is visualized via a dashboard tool for monitoring and follow-up of the ongoing experiments and easy detection of problems within the deployed installations. More complex analysis scripts run on the Data Server to check the quality of the received measurements over long periods and get insight in the offered flexibility over time, per appliance type, day of the week, etc., as well as detailed analyses of the conducted experiments. All this data processing is executed on a separate server to assure that the performance of the Live Server is not affected. For the same reason the Live Server only contains data for the last two weeks.

The Development \& Analysis server acts also as a mirror for the Live Server to take over in case of serious problems and to implement and test new features and interfaces during the reference measurement period and the first experiments. The Live Server and Development \& Analysis Server are hosted in a professional datacenter to guarantee a very high uptime.
TABLE I

OVERVIEW OF RECEIVED DATA AT THE END OF APRIL 2014 (SINCE APRIL 2010) IN THE PILOT BACKEND.

\begin{tabular}{|c|c|c|}
\hline Data & Frequency & \# records \\
\hline $\begin{array}{l}\text { Consumption and production } \\
\text { measurements (household and } \\
\text { appliances) }\end{array}$ & $\begin{array}{ll}\text { Every } & 15 \\
\text { minutes } & \end{array}$ & 175 million \\
\hline $\begin{array}{l}\text { Metadata from smart } \\
\text { appliances (configuration } \\
\text { time, deadline, estimated } \\
\text { power train, selected program, } \\
\text { device status, actual start time, } \\
\text { start type, state of charge, } \\
\text { temperature, etc.) }\end{array}$ & $\begin{array}{l}\text { On every } \\
\text { relevant state } \\
\text { change }\end{array}$ & 19.7 million \\
\hline $\begin{array}{l}\text { Power and voltage measure- } \\
\text { ments for users with a smart } \\
\text { meter }\end{array}$ & $\begin{array}{l}\text { Every } \\
\text { minutes }\end{array}$ & 13.3 million \\
\hline $\begin{array}{l}\text { Online/offline info for home } \\
\text { gateway, smart plugs and } \\
\text { smart appliances }\end{array}$ & $\begin{array}{l}\text { Every } \\
\text { minutes }\end{array}$ & 169 million \\
\hline $\begin{array}{l}\text { Transformer measurements } \\
\text { (voltage, current, cos } \varphi, \\
\text { temperature per phase and per } \\
\text { feeder) }\end{array}$ & $\begin{array}{l}\text { Every } \\
\text { minutes }\end{array}$ & 4.2 million \\
\hline
\end{tabular}

Web Services Security (WS-Security [16]) is used to secure the communication between the servers of the ESPs and Flex Aggregators and the Linear backend. Clients are required to encrypt all traffic using certificates issued by our Public Key Infrastructure (PKI). This way a system is created with authenticated hosts, exchanging confidential, non-repudiable messages. All servers are protected by a firewall and the dashboard tool can only be used by selected researchers and administrators with an account. The connection between the data server and a researcher who wants to perform a query is secured using secure shell (SSH). Data replication and synchronization between the different servers is done over SSH tunnelled connections.

\section{EXPERIMENTAL RESUlTS}

From the installation of the first measurement devices (April 2010) until April 2014 (with still 6 months of field trial ahead) more than 381 million records are gathered in the database of the pilot backend. Table I gives an overview of the different types of data and respective amounts that are exchanged between the households and the central database.

For monitoring the incoming data in real time, follow-up of running experiments and inspecting individual setups a dashboard tool was developed which visualizes the captured data both per individual user and aggregated over groups of users and appliance types. As an example, Figure 5 shows the quarterly energy measurements for one household for one day. The tool can show similar graphs for the flexibility data generated by the smart appliances and extra measurements of the smart meters (power, voltage), as well as differences between expected and actual number of received records which 


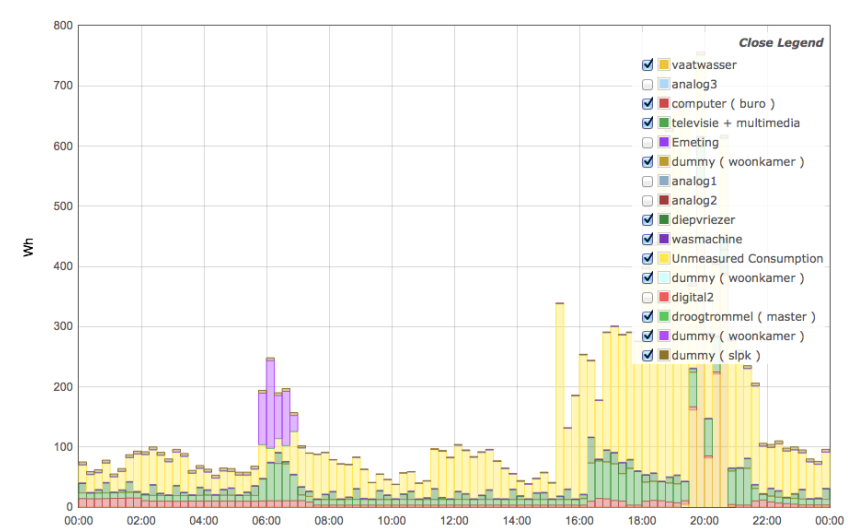

Fig. 5. Detailed consumption measurements for a single household for one day.

can help to detect problems. This dashboard tool has its own database that is fed by the aggregation scripts on the Data Server. As such it contains only the data that needs to be visualized which improves responsiveness.

Figure 6 shows a detailed analysis of the shifted power consumption of a household for the Portfolio Management business case when flexibility is provided (in this case by a dishwasher that is programmed around $17 \mathrm{~h} 30$ in the evening with flexibility until $3 \mathrm{~h} 15$ at night). The dishwasher is started at midnight when the time of use tariff is minimal (green bars) instead of at $17 \mathrm{~h} 30$ as it would have been without flex (red bars). This has a clear effect on the total household consumption (green line vs red dashed line without flex) with a smaller peak in the evening and considerably more consumption during the night.

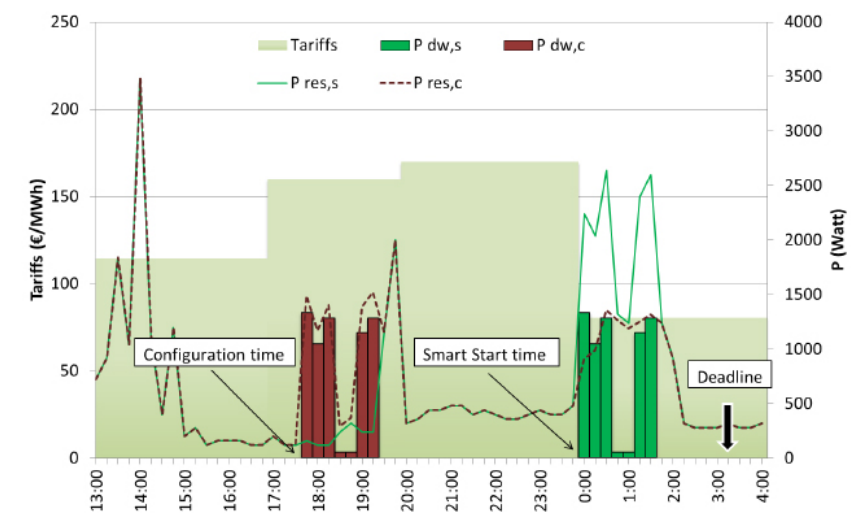

Fig. 6. Example analysis of the shifted power consumption of a household when flexibility is provided for the dishwasher (Portfolio Management case).

\section{CONCLUSIONS}

In this paper we presented the large-scale pilot on residential demand response of the Linear project, with main focus on the design and implementation of the supporting ICT architecture.

During the course of the pilot, it has become clear that one of the key problems why it has proven difficult to bridge from pilots to full scale rollout is interoperability, more specifically at following three levels: smart device abstraction, support of multiple parallel providers with proprietary and differing control technology and concurrent support of balancing and congestion management business cases for BRP, TSO and DSO. Because of the wide scope of the Linear pilot, interoperability was an important design criterion for its architecture (see Section III) and as a consequence the Linear architecture successfully addressed above issues, safe for the support of concurrent business cases.

At this moment the first profound analyses of the conducted experiments are performed. By the end of 2014 we will have a good view on the flexibility potential of residential customers and economic viability of the different researched business cases.

\section{ACKNOWLEDGMENT}

This work is supported by the Ministry of Science (Minister I. Lieten) via the project Linear organized by the Institute for Science and Technology (IWT).

\section{REFERENCES}

[1] European Parliament, "EU climate and energy package," http://ec.europa.eu/clima/policies/package/docs/climate_package_en.pdf, 2009.

[2] A. Ipakchi and F. Albuyeh, "Grid of the future," Power and Energy Magazine, IEEE, vol. 7, no. 2, pp. 52 -62, march-april 2009.

[3] R. Belhomme, R. Cerero, G. Valtorta, and P. Eyrolles, "The address project: Developing active demand in smart power systems integrating renewables," in Power and Energy Society General Meeting, 2011 IEEE, July 2011, pp. 1-8.

[4] A. Faruqui and S. Sergici, "Household response to dynamic pricing of electricity: a survey of 15 experiments," Journal of Regulatory Economics, vol. 38, no. 2, pp. 193-225, 2010.

[5] K. Herter, Y. Okunevo, and V. Wood, "Smud's residential summer solutions, ibc," Herter Energy Solutions, Tech. Rep., January 2014.

[6] R. Kamphuis, B. Roossien, M. Eijgelaar, H. de Heer, J. van de Velde, and A. van den Noort, "Real-time trade dispatch of a commercial VPP with residential customers in the powermatching city smartgrid living lab," in Electricity Distribution (CIRED 2013), 22nd International Conference and Exhibition on, June 2013, pp. 1-4.

[7] S. Lannez, G. Foggia, M. Muscholl, J. Passelergue, C. Lebosse, and K. Mercier, "Nice grid: Smart grid pilot demonstrating innovative distribution network operation," in IEEE PowerTech (POWERTECH) Grenoble, June 2013, pp. 1-5.

[8] H. Saele and O. Grande, "Demand response from household customers: Experiences from a pilot study in norway," Smart Grid, IEEE Transactions on, vol. 2, no. 1, pp. 102-109, March 2011.

[9] C. Woo, R. Li, A. Shiu, and I. Horowitz, "Residential winter kwh responsiveness under optional time-varying pricing in British Columbia," Applied Energy, vol. 108, no. C, pp. 288-297, 2013.

[10] "Toyota city low-carbon society verification project," http://jscp.nepc.or.jp/en/toyota.

[11] "Ecogrid EU: From design to implementation," http://ecogridbornholm.dk/wp-content/uploads/2013/10/EcoGridReport-Octobre-2013.pdf, October 2013.

[12] "Your energy moment project (jouw energie moment)," http://jouwenergiemoment.nl/.

[13] "Greenlys project," http://www.greenlys.fr/.

[14] B. Dupont, J. Tant, and R. Belmans, "Automated residential demand response based on dynamic pricing," in Innovative Smart Grid Technologies (ISGT Europe), 2012 3rd IEEE PES International Conference and Exhibition on, Oct 2012, pp. 1-7.

[15] K. Vanthournout, R. D'hulst, D. Geysen, and G. Jacobs, "A smart domestic hot water buffer," Smart Grid, IEEE Transactions on, vol. 3, no. 4, pp. 2121-2127, 2012.

[16] OASIS - Advancing open standards for the information society, "Web services security," https://www.oasis-open.org/standards\#wssv1.1.1. 